ISSN 0258-7122 (Print), 2408-8293 (Online)

Bangladesh J. Agril. Res. 42(2): 221-232, June 2017

\title{
ASSESSMENT OF ARBUSCULAR MYCORRHIZAL ASSOCIATION IN SOME FRUIT AND SPICE PLANTS OF RANGAMATI HILL DISTRICT
}

\author{
M. A. H. BHUIYAN ${ }^{1}$, M. B. BANU ${ }^{2}$ AND M. RAHMAN ${ }^{3}$
}

\begin{abstract}
Rhizosphere soils of some fruit and spice plants from the Hill Agricultural Research Station, Bangladesh Agricultural Research Institute, Raikhali, Rangamati were collected during 2011-12 and 2012-13 for counting Arbuscular Mycorrhizal (AM) spore population, determining colonization (\%) in their roots and studying AM structure. Assessment of spore population was done by following the Wet Sieving and Decanting Method. The percentage of AM infection was estimated by root slide technique. The spore number of $100 \mathrm{~g}$ rhizosphere soil was recorded ranging from 120 in rhizosphere soil of Malta plant to a maximum of 410 in Atafal and Sofeda plants during 2011-12 and from 75 in rhizosphere soil of Phalsa plant to a maximum of 327 in Amlaki plant during 2012-13. Different fruit and spice plants showed different percentages of root colonization by AM fungi. Among the fruit and spice plants, the highest colonization (40\%) was found in Jabotica, Phalsa and Sofeda plant, and the lowest colonization (6.6\%) was found in Rambutan plant during 2011-12, but in 2012-13 the highest (61.3\%) was result was observed in Bilatigab plant and the lowest $(18.7 \%)$ was in Misti lebu, Malta and Tetul plant. The AM fungal structure in the root system of the selected fruit and spice plants varied in irrespective of fruit and spice species. Some plants had vesicles. Hyphae were present in most of the plants. Some plant species recorded Arbuscules. Both oval and spherical shape vesicles were found in this study.
\end{abstract}

Keywords: Arbuscular mycorrhiza, spore population, root colonization, fruit \& spice plants

\section{Introduction}

Mycorrhizae are symbiotic association between beneficial soil fungi and plant roots. Vesicular-arbuscular mycorrhizae (VAM) are the mycorrhizae of crop plants and most annual and woody natives. They do not produce visible mushroom-type reproductive structures, but form spores that are the largest of any fungi. They cannot be grown in laboratory conditions, but they can grow with a wide variety of host plants. They have an important role in increasing plant uptake of poorly mobile nutrients such as $\mathrm{P}, \mathrm{Zn}$ and $\mathrm{Cu}$ (O'Keefe and Sylvia, 1991). Mycorrhizal plants are more resistant to some pathogens, have altered production of plant hormones, and have more highly branched root systems than non-mycorrhizal plants. Cuttings of some species have an improved

${ }^{1}$ Principal Scientific Officer, Soil Microbiology Laboratory, Bangladesh Agricultural Research Institute (BARI), Gazipur-1701, Bangladesh, ${ }^{2 \& 3}$ Scientific Officer, Soil Microbiology Laboratory, BARI, Gazipur-1701, Bangladesh. 
rooting ability when the medium contains mycorrhizal fungi. Many of the benefits, even those which seem unrelated to phosphorus, appear to actually be side benefits of improved phosphorus nutrition. The soil, as well as the plants, is affected by mycorrhizal fungi. The hyphae are an important component of soil structure, holding together crumbs that allow penetration of water and air, and encourage the growth of roots through the soil. Out of the different types of mycorrhizae, the AM fungi are the most widely occurring mycorrhizae and are very important in relation to the improvement of agricultural and horticultural crops and forest trees in hilly areas (Mridha and $\mathrm{Xu}, 2001$ ).

Arbuscular mycorrhizal fungi (AMF) that form symbiotic relationships with the roots of most terrestrial plants are known to improve the nutritional status of their host and to protect plants against several soil-borne plant pathogens (Smith and Read, 1997; Harrison, 1999; Bi et al., 2007). The major effect of mycorrhizal fungi in undisturbed ecosystems is to improve by the growth of mycorrhizal plants compared to non-mycorrhizal plants (Plenchette et al., 1983). It covers the root of plants so it makes protective physical barrier against diseases also (McAllister et al., 1997; Karagiannidis et al., 2002).

There are many disease management methods such as crop rotation, use of resistant varieties and chemical pesticides. However, frequent and indiscriminate use of these pesticides affects the physical, chemical and biological property of the soil. It also affects the non-target organisms and has developed resistance among the pathogen against these chemicals (Arwry and Quandt, 2003). Biocontrol potential of AM fungi against various phytopathogens is well documented (Singh et al., 2000; Kasiamdari et al., 2002; Azcón-Aguilar et al., 2002; Bodker et al., 2002; Xavier and Boyetchko, 2014; St-Arnaud and Elsen, 2005; St-Arnaud and Vujanovic, 2007). Arbuscular Mycorrhizal Fungi (AMF) are the major component of the rhizosphere of most of the plants and play a very important role as biocontrol agent and help in decreasing plant disease incidence (Akthar and Siddiqui, 2008).

They form three-way associations involving plants, fungi and soils. Bangladesh produces a variety of fruits and spices. It seems that there is an important role of arbuscular mycorrhizal fungi in nutrient availability for these fruit and spice plants. But still no work has been done to assess the mycorrhizal association with different fruit plants. So, this present work was undertaken to know the percent root colonization of fruit and spice plants and the number of AM spores in the rhizosphere soils for producing suitable inoculum for future use in different crops.

\section{Materials and Method}

Rhizosphere soils of some fruit and spice plants from the Hill Agricultural Research Station (HARS), Bangladesh Agricultural Research Institute (BARI), Raikhali, Rangamati were collected during 2011-12 and 2012-13 for the 
assessment of arbuscular mycorrhizal association. Rhizosphere soils with thin roots were collected from the plants, the list is presented in Table 1.

Table 1. List of plants collected from HARS, Raikhali, Rangamati during 2011-12 and 2012-13

\begin{tabular}{|c|c|c|c|}
\hline Local name & English name & Scientific name & Family \\
\hline Aam & Mango & Mangifera indica & Anacardiaceae \\
\hline Amlaki & Aonla & Phyllanthus emblica & Euphorbiaceae \\
\hline Amra & Hog plum & Spondias mangifera & Anacardiaceae \\
\hline Anar & Pomegranate & Punica granatum & Punicaceae \\
\hline Alubukhara & Pulm/Peach & Prunus persica & Rosaceae \\
\hline Arboroy & Star gooseberry & Phyllanthus acidus & Euphorbiaceae \\
\hline Ashfal & Longan & Nephelium longana & Sapindaceae \\
\hline Atafal & Bullock's heart & Annona reticulata & Annonaceae \\
\hline Avocado & Avocado & Persea americana & Lauraceae \\
\hline Batabilebu & Pummelo & Citrus grandis & Rutaceae \\
\hline Bael & Wood apple/Bael & Aegle marmelos & Rutaceae \\
\hline Bilatigab & Velvet apple & Diospyros discolor & Ebenaceae \\
\hline Bilimbi & Bilimbi & Averrhoa bilimbi & Oxalidaceae \\
\hline Bokful & Heron flower & Sesbania grandiflora & Fabaceae \\
\hline Chalta & Indian dillenta & Dillenia indica & Dilleniaceae \\
\hline Cherryfal & Cherry & Prunus avium & Rosaceae \\
\hline Coco & Cocoa & Theobroma cocoa & Malvaceae \\
\hline Cowfal & Cowa & Garcinia cowa & Clusiaceae \\
\hline Dalim & Pome granate & Punica granatum & Punicaceae \\
\hline Dewa & Monkey jackfruit & Artocarpus lakoocha & Moraceae \\
\hline Gab (Deshi) & River ebony & Diospyros peregrina & Ebenaceae \\
\hline Golapjam & Rose apple & Syzygium jambos & Myrtaceae \\
\hline Jabotica & Jabuticaba & Myrciaria cauliflora & Myrtaceae \\
\hline Jalpai & Indian oliva & Olea europaea & Oleraceae \\
\hline Jam & Jamun & Syzygium cumini & Myrtaceae \\
\hline Jamrul & Wax jambu & Syzygium samarengense & Myrtaceae \\
\hline Jamir & Rough lemon & Citrus jambhiri & Rutaceae \\
\hline Jilapifal & Jungle jalebi & Pithecellobium dulce & Fabaceae \\
\hline Kamranga & Carambola & Averrhoa carambola & Oxalidaceae \\
\hline Karamcha (Misti) & Carunda (sweet) & Carissa carandas & Apocynaceae \\
\hline Khejur & Date palm & Phoenix dactylifera & Palmae \\
\hline Kodbel & Elephant apple & Feronia elephantum & Rutaceae \\
\hline Komola & Orange/Mandarin & Citrus reticulata & Rutaceae \\
\hline Lebu (Misti) & Sweet lime & Citrus limettioides & Rutaceae \\
\hline
\end{tabular}




\begin{tabular}{llll}
\hline \multicolumn{1}{c}{ Local name } & \multicolumn{1}{c}{ English name } & \multicolumn{1}{c}{ Scientific name } & \multicolumn{1}{c}{ Family } \\
\hline Lotkan & Burmese grape & Baccaurea sapida & Euphorbiaceae \\
Macadamia & Macadamia nut & Macadamia integrifolia & Proteaceae \\
Mahuafal & Butter fruit & Madhuka indica & Sapotaceae \\
Malta & Sweet orange & Citrus sinensis & Rutaceae \\
Panifal & Water chestnut & Trapa bispinosa & Trapaceae \\
Peachfal & Peach & Prunus persica & Rosaceae \\
Persimmon & Persimmon & Diospyros kaki & Ebenaceae \\
Peyara (Thai) & Guava & Psidium guajava & Myrtaceae \\
Peyara (Seedless) & Guava & Psidium guajava & Myrtaceae \\
Phalsa & Phalsa/Dhamani & Grewia asiatica & Tilaiaceae \\
Rambutan & Rambutan & Nephelium lappaceum & Sapindaceae \\
Sajna & Drumstick & Moringa oleifera & Moringaceae \\
Satkara & Satkara & Citrus macroptera & Rutaceae \\
Sharifa & Custard apple & Annona squamosa & Annonaceae \\
Sofeda & Sapota & Manilkara achras & Sapotaceae \\
Tarokafal & Star apple & Chrysophyllum cainito & Sapotaceae \\
Tetul & Tamarind & Tamarindus indica & Fabaceae \\
Tuthfal & Mulberry & Morus indica & Moraceae \\
\hline & & & \\
\hline
\end{tabular}

\section{Assessment of spore population density}

Assessment of spore population was done by following the Wet Sieving and Decanting Method (Gerdemann and Nicolson, 1963). Soil samples from the rhizosphere of the respective plant species were mixed thoroughly by breaking up any large lumps. Any large unwanted particles such as stone, roots, twigs etc. were removed. Then $100 \mathrm{~g}$ of mixed soil was kept in a series of buckets (8-litre) and filled three quarters with tap water. The soil with water was agitated by stirring vigorously by hand and left to settle for some minutes. Two sieves (400 $\mu \mathrm{m}$ and $100 \mu \mathrm{m}$ mesh) were used throughout the experiment. The supernatant was poured through a $100 \mu \mathrm{m}$ sieve into the second bucked (10-litre) to avoid the loss of useful materials. The supernatant was decanted into the $400 \mu \mathrm{m}$ sieve. This time water was discarded and the material was back washed from the sieve into a beaker $(250 \mathrm{~mL})$ with a small quantity of water. The solution with spores was distributed in 4 equal size test tubes evenly and balanced up the tubes with water for equal weight. The tubes were plugged properly and then centrifuged for 4 minutes at 3,000 rpm. The supernatant was poured in test tubes and the test tubes were filled with sucrose solution and stirred vigorously with the roundended spatula to re-suspend the precipitate. The plugged test tubes were centrifuged for 15 seconds at 3,000 rpm. After centrifuge, the sucrose supernatant was poured through a $400 \mu \mathrm{m}$ sieve and rapidly washed with water to remove the 
sucrose from AM spores by back washing the materials from the sieve into watch glass for observation.

\section{Counting of AM spores}

All the AM spores were isolated from the extract with the help of a fine forcep into a watch glass with small quantity of water. The extract, with AM spores, was observed under stereomicroscope and the number of spores was counted. Spore numbers from the three replicates per samples were averaged and the result was expressed as number per $100 \mathrm{~g}$ of dry soil basis.

\section{Assessment of root colonization infection}

The percentage of AM infection was estimated by root slide technique (Read et al., 1976). One hundred root segments were examined for each sample. The stained root pieces were mounted in acidic glycerol on slides and the cover slip was placed, and slightly pressed. The roots were observed under microscope. A root segment was considered as positively infected, if it showed mycelium, vesicles and arbuscules or any other combination of these structural characteristics of AM infection. The presence or absence of infection in the root pieces was recorded and the percent infection was calculated as follows:

$\%$ root colonization $=\frac{\text { Number of AM positivesegments }}{\text { Total number of segments observed }} \times 100$

\section{Methods of chemical analysis:}

Soil pH was measured by a combined glass calomel electrode (Jackson, 1958). Organic carbon was determined by wet oxidation method (Page et al., 1982). Total $\mathrm{N}$ was determined by modified Kjeldahl method (Page et al., 1982). Calcium, $\mathrm{K}$ and $\mathrm{Mg}$ were determined by $\mathrm{NH}_{4} \mathrm{OAc}$ extraction method. Copper, $\mathrm{Fe}$, $\mathrm{Mn}$ and $\mathrm{Zn}$ were determined by DTPA extraction followed by AAS reading. Boron was determined by $\mathrm{CaCl}_{2}$ extraction method. Phosphorus was determined by Bray and Kurtz method. Sulphur was determined by $\mathrm{CaH}_{4}\left(\mathrm{PO}_{4}\right)_{2} \cdot \mathrm{H}_{2} \mathrm{O}$ extraction followed by turbidimetric turbidity method with $\mathrm{BaCl}_{2}$. The soil had $\mathrm{pH}$ value 5.7 with organic matter $1.83 \%$, exchangeable $\mathrm{Ca} 6.0$ meq $100 \mathrm{~g}^{-1}$, exchangeable $\mathrm{Mg} 2.1 \mathrm{meq} 100 \mathrm{~g}^{-1}$, exchangeable $\mathrm{K} .18$ meq $100 \mathrm{~g}^{-1}$, Total $\mathrm{N}$ $0.10 \%$, available P $16 \mu \mathrm{g} \mathrm{g}^{-1}$, available $\mathrm{S} 12 \mu \mathrm{g} \mathrm{g}^{-1}$, available B $0.16 \mu \mathrm{g} \mathrm{g}^{-1}$, available $\mathrm{Cu} 1.90 \mu \mathrm{g} \mathrm{g}^{-1}$, available $\mathrm{Fe} 48 \mu \mathrm{g} \mathrm{g}^{-1}$, available $\mathrm{Mn} 10.4 \mu \mathrm{g} \mathrm{g}^{-1}$, available $\mathrm{Zn} 2.87 \mu \mathrm{g} \mathrm{g}^{-1}$.

\section{Results and Discussion}

\section{Spore population of AM fungi}

Arbuscular mycorrhizal spores were assessed from different fruit and spice plants (Tables 2 and 3). During 2011-12, the highest spore number (410 per $100 \mathrm{~g}$ soil) 
was obtained from the rhizosphere soil of Atafal and the lowest spore number (120 per $100 \mathrm{~g}$ soil) was obtained from Malta plant (Table 2). Some of the fruit and spice plants like Anar, Atafal, Bael, Bilimbi, Jam, Jamrul, Jilapifal, Kamranga, Khejur, Peyara (Thai), Rambutan, Sajna, Sofeda and Tetul plants recorded more than 300 spores per $100 \mathrm{~g}$ rhizosphere soil during the first year (2011-12). During 2012-13, the highest spore number (327 per $100 \mathrm{~g}$ soil) was obtained from the rhizosphere soil of Amlaki plant and the lowest spore number (75 per $100 \mathrm{~g}$ soil) was obtained from Phalsa plant (Table 3). Some of the plants like Amlaki, Cherryfal and Lotkan plant recorded more than 300 spores per $100 \mathrm{~g}$ rhizosphere soil during the second year. There were wide variations in shape, size and colour of spores. Different shape and sizes of spore like round, oval, spherical etc. were found among the rhizosphere soils of different fruit and spice plants. In case of colours, like deep brown, light brown, radish, black, etc. were recorded in rhizosphere soils of different fruit and spice plants.

Table 2. Spore population of arbuscular mycorrhizae in rhizosphere soil and root infection of different fruit and spices plants of HARS, Raikhali, Rangamati during 2011-12.

\begin{tabular}{|c|c|c|c|c|c|c|c|}
\hline \multirow[b]{2}{*}{ Local name } & \multirow[b]{2}{*}{ English name } & \multirow{2}{*}{$\begin{array}{l}\text { Spore number } \\
\text { per } 100 \mathrm{~g} \mathrm{soil}^{\mathrm{a}}\end{array}$} & \multirow{2}{*}{$\begin{array}{c}\text { Root } \\
\text { colonization }^{\mathrm{a}} \\
(\%)\end{array}$} & \multicolumn{4}{|c|}{ AM structure ${ }^{b}$} \\
\hline & & & & $\mathrm{H}$ & A & $\mathrm{V}$ & VS \\
\hline Aam & Mango & $205.0 \pm 8.7$ & $10.0 \pm 1.2$ & + & + & - & - \\
\hline Amlaki & Aonla & $185.0 \pm 11.5$ & $9.3 \pm 1.2$ & + & - & - & - \\
\hline Amra & Hog plum & $280.0 \pm 11.5$ & $38.0 \pm 4.2$ & + & - & - & - \\
\hline Anar & Pomegranate & $322.0 \pm 12.7$ & $20.0 \pm 2.9$ & + & - & - & - \\
\hline Alubukhara & Pulm/Peach & $325.0 \pm 8.7$ & $10.0 \pm 1.2$ & + & - & - & - \\
\hline Arboroy & Star gooseberry & $210.0 \pm 11.5$ & $28.7 \pm 1.9$ & + & - & - & - \\
\hline Ashfal & Longan & $275.0 \pm 8.7$ & $20.0 \pm 2.3$ & + & - & + & $\mathrm{O}$ \\
\hline Atafal & Bullock's heart & $410.0 \pm 11.5$ & $10.0 \pm 1.2$ & + & - & - & - \\
\hline Avocado & Avocado & $250.0 \pm 8.7$ & $20.0 \pm 2.9$ & + & - & - & - \\
\hline Batabilebu & Pummelo & $270.5 \pm 11.5$ & $18.7 \pm 1.9$ & + & - & - & - \\
\hline Bael & Wood apple/Bael & $350.0 \pm 11.5$ & $20.0 \pm 2.9$ & + & - & - & - \\
\hline Bilatigab & Velvet apple & $195.0 \pm 8.7$ & $20.0 \pm 2.9$ & + & - & - & - \\
\hline Bilimbi & Bilimbi & $320.0 \pm 11.5$ & $18.7 \pm 4.1$ & + & - & - & - \\
\hline Bokful & Heron flower & $170.0 \pm 8.7$ & $20.0 \pm 2.9$ & + & - & - & - \\
\hline Chalta & Indian dillenta & $180.0 \pm 11.5$ & $10.7 \pm 1.2$ & + & - & - & - \\
\hline Cherryfal & Cherry & $140.0 \pm 8.7$ & $9.3 \pm 0.6$ & + & + & - & - \\
\hline Coco & Cocoa & $195.0 \pm 8.7$ & $6.9 \pm 1.2$ & + & - & - & - \\
\hline Cowfal & Cowa & $265.0 \pm 8.7$ & $30.0 \pm 2.9$ & + & - & - & - \\
\hline Dalim & Pome granate & $225.0 \pm 14.4$ & $20.0 \pm 2.9$ & + & - & - & - \\
\hline Dewa & Monkey jackfruit & $290.0 \pm 17.3$ & $10.0 \pm 1.2$ & + & - & - & - \\
\hline Gab (Deshi) & River ebony & $210.0 \pm 5.8$ & $30.0 \pm 2.9$ & + & - & - & - \\
\hline
\end{tabular}




\begin{tabular}{|c|c|c|c|c|c|c|c|}
\hline \multirow[b]{2}{*}{ Local name } & \multirow[b]{2}{*}{ English name } & \multirow{2}{*}{$\begin{array}{l}\text { Spore number } \\
\text { per } 100 \mathrm{~g} \mathrm{soil}^{\mathrm{a}}\end{array}$} & \multirow{2}{*}{$\begin{array}{c}\text { Root } \\
\text { colonization }^{\mathrm{a}} \\
(\%)\end{array}$} & \multicolumn{4}{|c|}{ AM structure ${ }^{b}$} \\
\hline & & & & $\mathrm{H}$ & A & $\mathrm{V}$ & VS \\
\hline Golapjam & Rose apple & $180.0 \pm 5.8$ & $20.0 \pm 2.9$ & + & - & - & - \\
\hline Jabotica & Jabuticaba & $185.0 \pm 14.4$ & $40.0 \pm 5.8$ & + & - & - & - \\
\hline Jalpai & Indian oliva & $195.0 \pm 8.7$ & $28.7 \pm 3.2$ & + & - & - & - \\
\hline Jam & Jamun & $320.0 \pm 11.5$ & $21.6 \pm 0.9$ & + & - & - & - \\
\hline Jamrul & Wax jambu & $320.0 \pm 17.3$ & $10.0 \pm 1.2$ & + & - & - & - \\
\hline Jamir & Rough lemon & $250.0 \pm 5.8$ & $28.7 \pm 3.2$ & + & + & + & $\mathrm{S}$ \\
\hline Jilapifal & Jungle jalebi & $300.0 \pm 28.9$ & $20.0 \pm 2.3$ & + & - & - & - \\
\hline Kamranga & Carambola & $325.0 \pm 14.4$ & $18.7 \pm 2.2$ & - & + & - & - \\
\hline $\begin{array}{l}\text { Karamcha } \\
\text { (Misti) }\end{array}$ & Carunda (sweet) & $190.0 \pm 11.5$ & $10.0 \pm 0.6$ & + & - & + & $\mathrm{O}$ \\
\hline Khejur & Date palm & $321.7 \pm 14.8$ & $20.0 \pm 1.2$ & + & - & - & - \\
\hline Kodbel & Elephant apple & $210.0 \pm 5.8$ & $18.7 \pm 0.9$ & + & - & - & - \\
\hline Komola & Orange/Mandarin & $215.0 \pm 8.7$ & $28.7 \pm 1.3$ & + & - & - & - \\
\hline Lebu (Misti) & Sweet lime & $185.0 \pm 8.7$ & $28.3 \pm 1.2$ & + & - & - & - \\
\hline Lotkan & Burmese grape & $210.0 \pm 17.3$ & $28.7 \pm 0.9$ & + & - & - & - \\
\hline Macadamia & Macadamia nut & $190.0 \pm 17.3$ & $20.0 \pm 1.2$ & + & + & - & - \\
\hline Mahuafal & Butter fruit & $200.0 \pm 23.1$ & $20.0 \pm 1.7$ & + & - & - & - \\
\hline Malta & Sweet orange & $120.0 \pm 8.7$ & $18.7 \pm 1.3$ & + & + & - & - \\
\hline Panifal & Water chestnut & $270.0 \pm 8.7$ & $20.0 \pm 1.7$ & + & - & - & - \\
\hline Peachfal & Peach & $230.0 \pm 5.8$ & $10.0 \pm 1.2$ & + & - & - & - \\
\hline Persimmon & Persimmon & $220.0 \pm 11.5$ & $10.0 \pm 1.2$ & + & - & + & $\mathrm{S}$ \\
\hline Peyara (Thai) & Guava & $300.0 \pm 23.1$ & $30.0 \pm 1.7$ & + & - & - & - \\
\hline $\begin{array}{l}\text { Peyara } \\
\text { (Seedless) }\end{array}$ & Guava & $251.7 \pm 41.1$ & $20.0 \pm 2.9$ & + & - & - & - \\
\hline Phalsa & Phalsa/Dhamani & $292.0 \pm 4.6$ & $40.0 \pm 2.9$ & + & - & - & - \\
\hline Rambutan & Rambutan & $318.0 \pm 6.9$ & $6.6 \pm 0.9$ & + & + & - & - \\
\hline Sajna & Drumstick & $300.0 \pm 17.3$ & $30.0 \pm 1.7$ & + & - & - & - \\
\hline Satkara & Satkara & $190.0 \pm 5.8$ & $18.3 \pm 3.3$ & + & - & - & - \\
\hline Sharifa & Custard apple & $172.0 \pm 4.6$ & $10.0 \pm 0.6$ & + & - & - & - \\
\hline Sofeda & Sapota & $410.0 \pm 11.5$ & $40.0 \pm 2.9$ & + & - & - & - \\
\hline Tarokafal & Star apple & $250.0 \pm 28.9$ & $20.0 \pm 1.7$ & + & - & - & - \\
\hline Tetul & Tamarind & $327.0 \pm 15.6$ & $18.7 \pm 1.7$ & + & - & - & - \\
\hline Tuthfal & Mulberry & $175.0 \pm 8.7$ & $30.0 \pm 1.7$ & + & - & - & - \\
\hline
\end{tabular}

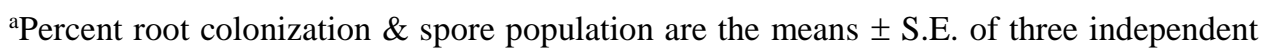
counts.

${ }^{\mathrm{b}} \mathrm{H}=$ Hypae, $\mathrm{A}=$ Arbuscle, $\mathrm{V}=$ Vesicle, $\mathrm{VS}=$ Vesicle Shape, $\mathrm{O}=\mathrm{Oval}, \mathrm{S}=$ Spherical 
Table 3. Spore population of arbuscular mycorrhizae in rhizosphere soil and root infection of different fruit and spices plants of HARS, Raikhali, Rangamati during 2012-13.

\begin{tabular}{|c|c|c|c|c|c|c|c|}
\hline \multirow[b]{2}{*}{ Local name } & \multirow[b]{2}{*}{ English name } & \multirow{2}{*}{$\begin{array}{l}\text { Spore number } \\
\text { per } 100 \mathrm{~g} \text { soil }{ }^{\mathrm{a}}\end{array}$} & \multirow{2}{*}{$\begin{array}{c}\text { Root } \\
\text { colonization }^{\mathrm{a}} \\
(\%)\end{array}$} & \multicolumn{4}{|c|}{ AM structure ${ }^{b}$} \\
\hline & & & & $\mathrm{H}$ & A & $\mathrm{V}$ & VS \\
\hline Aam & Mango & $266.0 \pm 22.5$ & $31.0 \pm 3.8$ & + & - & + & $\mathrm{O}$ \\
\hline Amlaki & Aonla & $327.0 \pm 1.7$ & $30.0 \pm 2.9$ & + & - & - & - \\
\hline Amra & Hog plum & $257.3 \pm 29.2$ & $20.0 \pm 2.9$ & + & - & - & - \\
\hline Anar & Pomegranate & $96.0 \pm 6.4$ & $20.7 \pm 3.5$ & + & - & - & - \\
\hline Alubukhara & Pulm/Peach & $140.3 \pm 14.1$ & $20.7 \pm 3.5$ & + & - & - & - \\
\hline Arboroy & Star gooseberry & $195.0 \pm 14.4$ & $20.0 \pm 2.9$ & + & - & - & - \\
\hline Ashfal & Longan & $200.0 \pm 17.3$ & $21.3 \pm 4.1$ & + & - & - & $\mathrm{O}$ \\
\hline Atafal & Bullock's heart & $175.0 \pm 17.3$ & $21.3 \pm 4.1$ & + & - & - & - \\
\hline Avocado & Avocado & $223.0 \pm 7.5$ & $20.0 \pm 2.9$ & + & - & - & - \\
\hline Batabilebu & Pummelo & $205.3 \pm 14.1$ & $20.0 \pm 2.9$ & + & - & - & - \\
\hline Bael & Wood apple/Bael & $190.0 \pm 23.0$ & $21.3 \pm 1.9$ & + & - & - & - \\
\hline Bilatigab & Velvet apple & $240 \pm 11.5$ & $61.3 \pm 4.1$ & + & - & - & $\mathrm{O}$ \\
\hline Bilimbi & Bilimbi & $92.3 \pm 7.2$ & $20.0 \pm 2.9$ & + & + & + & + \\
\hline Bokful & Heron flower & $155.3 \pm 11.8$ & $21.0 \pm 3.8$ & + & - & - & - \\
\hline Chalta & Indian dillenta & $198.3 \pm 4.9$ & $21.0 \pm 4.1$ & + & - & - & - \\
\hline Cherryfal & Cherry & $316.7 \pm 23.3$ & $20.0 \pm 2.9$ & + & + & + & $\mathrm{S}$ \\
\hline Coco & Cocoa & $82.3 \pm 7.2$ & $21.0 \pm 3.8$ & + & - & - & - \\
\hline Cowfal & Cowa & $156.0 \pm 17.9$ & $21.3 \pm 4.1$ & + & - & - & - \\
\hline Dalim & Pome granate & $262.3 \pm 38.9$ & $30.0 \pm 2.9$ & + & - & - & - \\
\hline Dewa & Monkey jackfruit & $287.3 \pm 47.6$ & $20.0 \pm 2.9$ & + & - & - & - \\
\hline Gab (Deshi) & River ebony & $134.7 \pm 35.0$ & $20.7 \pm 3.5$ & + & - & - & - \\
\hline Golapjam & Rose apple & $158.3 \pm 0.9$ & $21.0 \pm 3.8$ & + & - & - & - \\
\hline Jabotica & Jabuticaba & $125.0 \pm 2.9$ & $20.0 \pm 2.9$ & + & - & - & - \\
\hline Jalpai & Indian oliva & $291.7 \pm 20.5$ & $20.0 \pm 2.0$ & + & - & - & - \\
\hline Jam & Jamun & $217.3 \pm 7.2$ & $30.0 \pm 2.9$ & + & - & - & - \\
\hline Jamrul & Wax jambu & $215.3 \pm 26.0$ & $30.0 \pm 2.9$ & + & - & - & - \\
\hline Jamir & Rough lemon & $204.0 \pm 44.5$ & $20.0 \pm 2.9$ & + & - & - & - \\
\hline Jilapifal & Jungle jalebi & $89.3 \pm 8.3$ & $20.7 \pm 3.5$ & + & - & - & - \\
\hline Kamranga & Carambola & $155.0 \pm 1.2$ & $20.0 \pm 2.9$ & + & - & - & - \\
\hline $\begin{array}{l}\text { Karamcha } \\
\text { (Misti) }\end{array}$ & Carunda (sweet) & $280.0 \pm 5.8$ & $28.7 \pm 1.9$ & + & - & - & - \\
\hline Khejur & Date palm & $283.0 \pm 27.1$ & $21.0 \pm 3.8$ & + & - & - & $\mathrm{O}$ \\
\hline Kodbel & Elephant apple & $154.0 \pm 0.6$ & $28.7 \pm 1.9$ & + & - & - & - \\
\hline Komola & Orange/Mandarin & $122.3 \pm 21.7$ & $18.7 \pm 1.9$ & + & + & - & - \\
\hline
\end{tabular}




\begin{tabular}{|c|c|c|c|c|c|c|c|}
\hline \multirow[b]{2}{*}{ Local name } & \multirow[b]{2}{*}{ English name } & \multirow{2}{*}{$\begin{array}{l}\text { Spore number } \\
\text { per } 100 \mathrm{~g} \mathrm{soil}^{\mathrm{a}}\end{array}$} & \multirow{2}{*}{$\begin{array}{c}\text { Root } \\
\text { colonization }^{\text {a }} \\
(\%) \\
\end{array}$} & \multicolumn{4}{|c|}{ AM structure ${ }^{b}$} \\
\hline & & & & $\mathrm{H}$ & A & V & VS \\
\hline Lebu (Misti) & Sweet lime & $310.0 \pm 11.5$ & $20.0 \pm 2.9$ & + & - & - & - \\
\hline Lotkan & Burmese grape & $239.0 \pm 19.6$ & $18.7 \pm 1.9$ & + & - & - & - \\
\hline Macadamia & Macadamia nut & $188.3 \pm 10.7$ & $20.0 \pm 2.9$ & + & - & - & $\mathrm{O}$ \\
\hline Mahuafal & Butter fruit & $202.3 \pm 4.3$ & $30.0 \pm 2.9$ & + & - & & - \\
\hline Malta & Sweet orange & $215.0 \pm 31.8$ & $18.7 \pm 1.9$ & + & - & - & - \\
\hline Panifal & Water chestnut & $188.3 \pm 18.2$ & $20.0 \pm 2.9$ & + & - & - & - \\
\hline Peachfal & Peach & $220.0 \pm 5.8$ & $30.0 \pm 2.9$ & + & - & - & - \\
\hline Persimmon & Persimmon & $167.3 \pm 4.3$ & $21.3 \pm 4.1$ & + & - & - & - \\
\hline Peyara (Thai) & Guava & $108.3 \pm 10.7$ & $30.0 \pm 2.9$ & + & - & - & - \\
\hline $\begin{array}{l}\text { Peyara } \\
\text { (Seedless) }\end{array}$ & Guava & $182.3 \pm 13.0$ & $20.0 \pm 2.9$ & + & - & - & - \\
\hline Phalsa & Phalsa/Dhamani & $75.0 \pm 11.5$ & $21.3 \pm 4.1$ & + & - & - & $\mathrm{O}$ \\
\hline Rambutan & Rambutan & $97.3 \pm 4.3$ & $30.0 \pm 2.9$ & + & - & - & - \\
\hline Sajna & Drumstick & $131.3 \pm 2.6$ & $20.0 \pm 2.9$ & + & - & - & - \\
\hline Satkara & Satkara & $290.3 \pm 11.6$ & $28.7 \pm 1.9$ & + & - & - & - \\
\hline Sharifa & Custard apple & $160.3 \pm 12.7$ & $20.0 \pm 2.9$ & + & - & - & - \\
\hline Sofeda & Sapota & $282.3 \pm 7.2$ & $20.0 \pm 2.9$ & + & - & + & S \\
\hline Tarokafal & Star apple & $101.3 \pm 3.8$ & $30.0 \pm 2.9$ & + & - & - & - \\
\hline Tetul & Tamarind & $186.7 \pm 19.3$ & $18.7 \pm 1.9$ & + & - & - & - \\
\hline Tuthfal & Mulberry & $166.0 \pm 22.5$ & $30.0 \pm 2.9$ & + & - & - & - \\
\hline
\end{tabular}

aPercent root colonization \& spore population are the means \pm S.E. of three independent counts.

${ }^{\mathrm{b}} \mathrm{H}=$ Hypae, A=Arbuscle, V=Vesicle, VS= Vesicle Shape, O=Oval, S=Spherical.

In the present study, the spore density in the rhizosphere soil varied in different fruit and spice plants which were supported by Howeler et al. (1987) who reported that the intensity of spore density varied on different factors like plant species and genera and nature of rhizosphere soil. Moreover, higher spore population was observed in some fruit and spice plants. The stimulating effects of organic matter, comparatively higher level of $\mathrm{N}$ and $\mathrm{P}$ might have created a favourable condition for the maximum sporulation of AM fungi in that particular field.

\section{Root colonization}

Different fruit and spice plants showed different percentages of root colonization by Arbuscular mycorrhizal fungi (Tables 2 and 3). During the first year study (2011-12), the root colonization varied from $6.6 \%$ to $40.0 \%$ (Table 2). More than $30 \%$ colonization was recorded with Amra, Cowfal, Gab, Jabotica, Peyara 
(Thai), Phalsa, Sajna, Sofeda and Tuthfal rhizospheres. The lowest colonization (6.6\%) was recorded in Rambutan plants. During the second year (2012-13), root colonization varied from $18.7 \%$ to $61.3 \%$ (Table 3). Similar to the first year, above $30 \%$ colonization was noted with Aam, Amlaki, Bilatigab, Dalim, Jam, Jalpai, Mahuafal, Peyara (Thai), Rambutan, Tarakafal and Tuthfal plants. Among them, the highest colonization (61.3\%) was found in Bilatigab plant. The lowest colonization (18.7\%) was recorded in Malta, Lebu (Misti) and Tetul plant. A large variation was observed in the colonization among different fruit and spice plants. This variation might be due to the differences in the structure of root system and P uptake (Hetrick et al., 1992) and also might be due to genetic variations (Mercy et al., 1990).

\section{AM structure}

The AM fungal structure in the root system of the selected fruit and spice plants varied in irrespective of fruit and spice species (Tables 2 and 3). There were some plants which had vesicles. Hyphae were present in most of the plants. Some plant species recorded Arbuscules. Both oval and spherical shape vesicles were found in this study, which was supported by Khanam et al. (2003, 2004).

Spore number and root colonization varied from plant to plant in the present study. But variations in spore number in different plants were not related to per cent root colonization which is in agreement with Khalil et al. (1992). As a wide range of host, fungal and environmental factors are known to influence AM formation and subsequent spore production; these two phenomena may not necessarily be related.

\section{Conclusion}

It is evident from the study that spore number and root colonization varied from plant to plant. Variations in spore numbers in different plants were not related to per cent root colonization. The AM fungal structure in the root system of the selected spices and fruit plants also varied in irrespective of fruit and spice species. A wide range of host, fungal and environmental factors might have influenced AM formation and subsequent spore production. Higher root colonization of fruit and spices plants and higher number of AM spores in the rhizosphere soils can be selected for producing suitable inoculum for future use.

\section{References}

Akthar, M. S. and Z. A. Siddiqui. 2008. Arbuscular mycorrhizal fungi as potential bioprotectants against plant pathogens. In: Mycorrhizae; Sustainable Agriculture and Forestry, Siddiqui, Z. A., M. S. Akhtar and K. Futai (eds.). Springer Netherlands, Dordrecht, the Netherlands.

Arwry, T. A. and S. A. Quandt. 2003. Pesticides at work and at home: Exposure of migrant farmworkers. Lancet. 362: 2021-2021. 
Azcón-Aguilar, C., M. C. Jaizme-Vega and C. Calvet. 2002. The contribution of Arbuscular Mycorrhizal Fungi to the control of soilborne plant pathogens. In: Gianinazzi, S., H. Schuepp, J. M. Barea and K. Haselwandter (eds). Mycorrhizal Technology in Agriculture. Birkhauser, Switzerland, Pp. 187-197.

Bi, H. H., Y. Y. Song and R. S. Zeng. 2007. Biochemical and molecular responses of host plants to mycorrhizal infection and their roles in plant defense. Allelopath. J. 29: 15-27.

Bodker, L., R. Kjoller, K. Kristensen and S. Rosendahl. 2002. Interactions between indigenous Arbuscular Mycorrhizal Fungi and Aphanomyces euteiches in fieldgrown pea. Mycorrhiza. 12: 7-12.

Gerdemann, J. W. and T. H. Nicolson. 1963. Species of mycorrhizal endogone species extracted from soil by wet sieving and decanting method. Trans. Brit. Mycol. Soc. 46: $235-246$.

Harrison, M. J. 1999. Molecular and cellular aspects of the arbuscular mycorrhizal symbiosis. Plant Mol. Biol. 50: 361-389.

Hetrick, B. A. D., G. W. T. Wilson and T. C. Todd. 1992. Relationship of mycorrhizal symbiosis, rooting strategy, and phenology among tallgrass prairie forbs. Can. J. Bot. 70: $1521-1528$.

Howeler, R. H., E. Seiverding and S. Saif. 1987. Practical aspects of mycorrhizal technology in some tropical crops and pastures. Plant Soil. 100: 249-283.

Jackson, M. L. 1958. Soil Chemical Analysis. Constable and Co. Ltd., London.

Karagiannidis, N., F. Bletsos and N. Stavropoulos. 2002. Effect of Verticillium wilt (Verticillium dahliae Kleb.) and mycorrhiza (Glomus mosseae) on root colonization, growth and nutrient uptake in tomato and eggplant seedlings. Scientia Horticulturae, 94(1-2): 145-156.

Kasiamdari, R. S., S. E. Smith, F. A. Smith and E. S. Scott. 2002. Influence of the mycorrhizal fungus, Glomus coronatum and soil phosphorus on infection and disease caused by binucleate Rhizoctonia and Rhizoctonia solani on mung bean (Vigna radiata). Plant Soil. 238: 235-244.

Khalil, S., T. E. Loynachan and H. S. McNabb. 1992. Colonization of soybean by mycorrhizal fungi and spore population in Iowa soils. Agron. J. 84: 832-836.

Khanam, D., A. R. M. Solaiman, M. A. U. Mridha and A. J. M. S. Karim. 2003. Arbuscular mycorrhizal fungi association with some agricultural crops grown in four agroecological zones of Bangladesh. Bangladesh J. Soil Sci. 27-28: 1-12.

Khanam, D., A. R. M. Solaiman and M. A. U. Mridha. 2004. Biodiversity of arbuscular mycorrhizal fungi in agricultural crops grown under different agro ecological zones of Bangladesh. Bull. Inst. Trop. Agr., Kyushu Univ. 27: 25-33.

McAllister, C. B., J. M. Garcia-Garrido, I. Garcia-Romera, A. Godeas and J. A. Ocampo. 1997. Interaction between Alternaria alternata or Fusarium equiseti and Glomus mosseae and its effects on plant growth. Plant Soil. 24(3): 301-305.

Mercy, M. A., G. Shivashankar and D. J. Bagyaraj. 1990. Mycorrhizal colonization in cowpea is host dependent and heritable. Plant Soil. 121: 292-294.

Mridha, M. A. U. and H. L. Xu. 2001. Nature Farming with vesicular arbuscular mycorrhizae in Bangladesh. J. Crop Prod. 3: 303-312. 
O'Keefe, D. M. and D. M. Sylvia. 1991. Mechanisms of the vesicular arbuscular mycorrhizal plant growth response. In: Handbook of Applied Mycology. D. K. Arora (ed.). Marcel Dekker, New York, pp. 35-54.

Plenchette, C., J. A. Fortin and V. Furlan. 1983. Growth responses of several plant species to mycorrhizae in a soil of moderate P fertility. I. Mycorrhizal dependency under field conditions. Plant Soil. 70: 199-209.

Page, A. L., R. H. Miller and D. R. Keeney. 1982. Methods of Soil Analysis. Part 2. $2^{\text {nd }}$ Ed. Amer. Soc. Agron., Inc., Medison, Wisconsin, USA.

Read, D. J., H. K. Koucheki and J. Hodgaon. 1976. Vesicular arbuscular mycorrhiza in natural vegetation systems. New Phytol. 77: 641-653.

Singh, A., J. Sharma, K. H. Rexer and A. Varma. 2000. Plant productivity determinants beyond minerals, water and light: Piriformospora indica - A revolutionary plant growth promoting fungus. Curr. Sci. 79: 1548-1554.

Smith, S. E. and D. J. Read. 1997. Mycorrhizal symbiosis. Academic Press, London.

St-Arnaud, M. and A. Elsen. 2005. Interaction of arbuscular mycorrhizal fungi with soilborne pathogens and non-pathogenic rhizosphere micro-organisms. In: Declerck, S., D. G. Strullu and J. A. Fortin (eds). In vitro culture of mycorrhizas. Springer, Heidelberg, pp. 217-223.

St-Arnaud, M. and V. Vujanovic. 2007. Effect of the arbuscular mycorrhizal symbiosis on plant diseases and pests. In: Hamel, C. and C. Plenchette (eds). Mycorrhizae in crop production: applying knowledge. Haworth, Binghampton, N.Y., USA, pp. 67122.

Xavier, L. J. C. and S. M. Boyetchko. 2014. Arbuscular mycorrhizal fungi in plant disease control. In: Arora, D. K. (ed). Fungal biotechnology in agricultural, food, and environmental applications. Dekker, New York, pp. 183-194. 